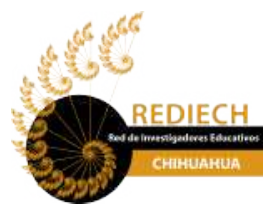

Red de Investigadores Educativos Chihuahua A.C. Chihuahua, México
ISSN: 2007-4336

ISSN-e: 2448-8550

http://www.rediech.org/ojs/2017/index.php/ie rie rediech/index

Jesús Adolfo Trujillo Holguín

2019

\title{
La investigación educativa y su vinculación con las necesidades sociales
}

IE Revista de Investigación Educativa de la REDIECH, 10(18), pp. 4-8. https://dx.doi.org/10.33010/ie rie rediech.v10i18.699

\section{(c) $(1)(\theta$}

Esta obra está bajo licencia internacional

Creative Commons Reconocimiento-NoComercial 4.0.

CC BY-NC 4.0 




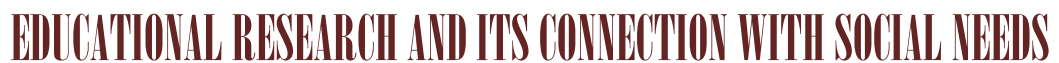

TRUJLLLO HOLGUÍN Jesús Adolfo ${ }^{1}$

lirentor

DOI: https://dx.doi.org/10.33010/ie_rie_rediech.v10i18.699

La actividad investigativa precisa no solamente del desarrollo de un proceso sistemático de interrogación y búsqueda de datos para comprobar supuestos, sino que obliga al investigador a que, una vez concluido su trabajo, encuentre los mecanismos para la difusión de resultados, de manera que sean útiles para la solución de problemáticas específicas; 0 bien, para que inciten a la generación de nuevas interrogantes que sean el principio de un nuevo proceso de indagación.

Desde hace unas cuantas décadas, cuando la investigación educativa en México se encontraba en proceso de gestación, el posgrado comenzó a visualizase como un espacio para la generación de conocimiento, aunque permeado con la idea de que el proceso investigativo concluía cuando los estudiantes presentaban la tesis y cuando los ejemplares impresos llegaban hasta el estante de una biblioteca. Poco a poco los investigadores educativos avanzaron en su carrera de profesionalización y en la adquisición de una identidad propia como comunidad científica, una vez que comenzaron a abrirse espacios de diálogo e interacción para la comunicación de resultados (Latapí, 2008).

Cuando los congresos y publicaciones académicas se consolidan como canales de comunicación de la ciencia, los investigadores dejaron de concebir la elaboración de productos (artículos, capítulos de libros, ponencias...) como simple placer para quienes estaban dotados de talento para la escritura y se convirtieron en un requisito más dentro del proceso natural de investigación. El Sistema Nacional de Investigadores del Consejo Nacional de Ciencia y Tecnología (SNI-Conacyt) impulsó dicha tendencia, otorgando el mayor valor en la evaluación de la productividad científica individual cuando esta lograba insertarse en revistas de alto impacto a nivel nacional e internacional.

La valoración de la calidad de la investigación con base en el factor de impacto de la revista donde se publica, llevó a que buena parte de los investigadores estuvieran más preocupados por compartir sus hallazgos con los lectores de una publicación indizada internacionalmente - preferentemente en las bases de datos de Scopus o Web of Science- que con los potenciales usuarios, ubicados quizás en la propia localidad de pertenencia. Se llegó a lo que Manuel Gil Antón señala como "escribir para ser evaluado, no para ser leído" $(2018, \mathrm{~s} / \mathrm{p})$.

La función social del investigador educativo va más allá de escribir para una revista académica prestigiada. Supone que mediante los productos generados se puedan:

${ }^{1}$ Universidad Autónoma de Chihuahua, México. Correo electrónico: jatrujillo@uach.mx. ID: http://orcid.org/0000-0002-6738-1878. 
[...] identificar problemas relevantes de su campo, derruir mitos, dimensionar complejidades o conceptuar de manera diferente una realidad cambiante. Al aportar nuevas visiones y generar un clima diferente para discutir aspectos relevantes de la problemática social, los investigadores de la educación contribuyen a la gestación de un ambiente cultural y un clima político que motive a un número creciente de personas a participar en la búsqueda de soluciones [López, Sañudo y Maggi, 2013, p. 21].

Bajo el supuesto anterior, la investigación educativa debe responder a las necesidades sociales, aunque a primera vista se antoje difícil conciliar las demandas del sector productivo y social con los intereses y motivaciones de los investigadores. A este respecto, el Conacyt ha realizado esfuerzos importantes por vincular las actividades de indagación con sus potenciales usuarios a través de la identificación de demandas específicas en los fondos concursables. Sin embargo, aún persiste el reto en la política pública para que los productos generados incidan en la toma de decisiones.

La experiencia en las reformas educativas recientes nos ha mostrado que los investigadores educativos y los tomadores de decisiones transitan por caminos distintos y en los documentos curriculares son escasas las referencias a trabajos de investigación que son ampliamente discutidos en congresos o difundidos en publicaciones académicas, aun y cuando sus autores gocen de notable prestigio académico en círculos como el Consejo Mexicano de Investigación Educativa (Casanova, 2018). Más lejos aún estamos de que los resultados de alguna investigación puedan traducirse en un lenguaje tan sencillo y claro que cualquier maestro frente a grupo pueda interpretarlo y llevarlo a la práctica en una situación concreta dentro de su ámbito laboral.

El pionero de la investigación educativa en México, Pablo Latapí Sarre, advirtió hace más de una década que debía buscarse la convergencia entre la investigación educativa y las necesidades sociales. Presentó como punto conclusivo, para augurar un mejor futuro en esta actividad que:

Nuestro conocimiento especializado debe llegar, en lenguajes adecuados, a la gran opinión pública, en busca no sólo de eficacia en las coyunturas inmediatas, sino de construir una opinión ciudadana mejor informada respecto de la educación. Junto con esto reforcemos los movimientos de la sociedad civil -en la cual radica aquella energía que califiqué como la principal para renovar la educación- para que esta sociedad se convierta en actor real, en sujeto reclamante de sus derechos, en contralor vigilante de las acciones gubernamentales y del desempeño de los maestros [Latapí, 2008, p. 295].

De esta manera, la investigación debe encaminarse en la búsqueda de soluciones concretas a los retos que tienen el sistema educativo y visualizar rutas alternativas que no se reduzcan exclusivamente a las publicaciones académicas. Los resultados de los procesos investigativos deben plasmarse en los libros de texto, en la opinión pública, en la capacitación de maestros y en todos los espacios donde sea posible el mejoramiento de los procesos educativos.

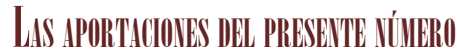

Aunque reconocemos que una publicación científica como IE Revista de Investigación Educativa de la Rediech por sí misma no puede incidir en una política pública, 
creemos que representa un espacio muy importante para cumplir con el mandato de acceso abierto que dispone la Ley de Ciencia y Tecnología de México. Mediante la publicación de los 14 trabajos que integran el presente número hacemos que "el conocimiento universal esté disponible, a texto completo y en formatos digitales a los educandos, educadores, académicos, investigadores, científicos, tecnólogos y población en general" (Congreso de la Unión, 2002, p. 35), siendo un insumo indispensable para el empoderamiento de la ciudadanía.

Este número inicia con el trabajo "Laboratorios reales versus laboratorios virtuales en las carreras de ciencias de la computación", donde Aníbal Zaldívar-Colado presenta los resultados de un estudio que tuvo por objeto determinar las preferencias de los estudiantes por los laboratorios reales 0 virtuales en las carreras de informática y ciencias de la computación. Los resultados sirven para analizar la manera en que la oferta educativa puede flexibilizarse para responder a las necesidades de los estudiantes, pues se hace evidente -por ejemplo- que quienes residen fuera de la ciudad donde se encuentra su institución educativa prefieren los laboratorios virtuales debido a las ventajas que representa acceder a ellos a cualquier hora y desde cualquier lugar mediante el uso de herramientas como el teléfono celular.

El artículo "Meta-habilidades en información y evolución conceptual en la educación virtual", de Felipe de Jesús Jasso Peña, José Porfirio Tamez Solís y José Antonio Torres Reyes, se ocupa de estudiar la habilidad para el acceso, uso y distribución de la información (meta-habilidades en información) y su papel en la evolución conceptual de estudiantes de posgrado virtual, encontrando que efectivamente existe una relación de estas con el desarrollo de conocimientos en los estudiantes.

Jorge Eduardo Martínez Íñiguez, Sergio Tobón y Evangelina López Ramírez muestran el tercer trabajo titulado "Currículo: un análisis desde un enfoque socioformativo", donde realizan un análisis documental apoyados en la teoría de la cartografía conceptual, para determinar cómo el currículo desde un enfoque socioformativo difiere de otras perspectivas por su énfasis en el trabajo con proyectos transversales que buscan contribuir al desarrollo social sostenible. Concluyen que es prioritario realizar diversos estudios que permitan transformar las prácticas educativas con enfoques que tomen en cuenta las características de la sociedad latinoamericana.

Bajo la misma línea del currículo, en el trabajo "Innovación y experiencias creativas de matemática educativa en escuelas secundarias desde la pedagogía del malabarismo", Pável Roel Gutiérrez Sandoval, Evangelina Cervantes Holguín e Iskra Rosalía Gutiérrez Sandoval analizan el área de las matemáticas mediante una propuesta innovadora basada en la pedagogía del malabarismo, donde infieren -luego de la aplicación de su propuesta- que los estudiantes mejoraron su rendimiento académico en temas de dicha asignatura.

El quinto trabajo se titula "Estado del arte sobre identidad docente: investigación de experiencias de profesores en formación y en ejercicio", de la autora chilena Karen Paulina Gajardo-Asbun. A través de una investigación documental, se expone que el tema de la identidad docente está vigente en varios países, pues revisa una veintena de investigaciones que buscan comprender las aristas de la identidad en los diversos contextos que vive el educador. A partir de los resultados, la autora espera contribuir con conocimiento de base para futuros estudios, los cuales den luz de lo que falta por explicar en este ámbito.

El artículo "El profesorado de una zona escolar ante el precepto de calidad educativa", de Luis Alan Acuña Gamboa y Leticia Pons Bonals, analiza las posiciones que el profesorado de escuelas primarias de una zona escolar ubicada en Chiapas 
(México) asumen frente al precepto de calidad educativa que se distribuye a través de los documentos oficiales que rigen su trabajo. Con base en la teoría de los capitales, propuesta por Bourdieu, y utilizando el enfoque cualitativo, se descubre que este profesorado se contrapone a los lineamientos de la política educativa centrados en el logro de la calidad educativa, porque estos se alejan de su contexto de aplicación, algo que reiteradamente se señaló en diversos sectores al impulsarse la reforma constitucional en materia educativa de 2013.

De la misma región sureste de México tenemos el noveno artículo titulado "Análisis del flujo de la matrícula escolar en primarias y secundarias: el caso del estado de Yucatán”, de Wilson Jesús Pool Cibrián, Felipe de la Cruz Lugo Lugo y William Eliezer Pacheco Ceballos. Los autores identifican el flujo de la matrícula escolar de varias generaciones de estudiantes para ofrecer indicadores de eficiencia terminal de la educación básica en el estado de Yucatán. Los resultados de este trabajo tienen implicaciones a nivel local, dado que permite identificar a estudiantes en riesgo de rezago educativo, haciendo posible que supervisores de zona y directores de escuela realicen acciones específicas en sus centros educativos.

En el rubro de análisis del campo de la investigación educativa aparecen dos artículos más. En el primero, Isabel Izquierdo Campos y Magali Atristan Hernández presentan "Experiencias de investigadoras en su ingreso, promoción y permanencia en el Sistema Nacional de Investigadores: tensiones y estrategias identitarias", donde abordan las vivencias de investigadoras de las ciencias en su ingreso, promoción y permanencia en el SNI. Se analizaron las tensiones que experimentaron y las estrategias identitarias que pusieron en marcha en dicho proceso, utilizando como herramienta el método biográfico. En el trabajo "Fundamentos teórico-metodológicos en la investigación educativa en Chihuahua, análisis de un área del conocimiento", Francisco Alberto Pérez Piñón, Stefany Liddiard Cárdenas y Guillermo Hernández Orozco nos muestran las tendencias teóricas y metodológicas investigativas del área denominada "Teoría, filosofía, historia e historiografía de la educación e investigación sobre la investigación educativa". Mediante el análisis de las ponencias presentadas en dos congresos de investigación educativa organizados por la Red de Investigadores Educativos Chihuahua, realizan un acercamiento teórico centrado en los paradigmas de la investigación histórica (historicismo, escuela francesa de los annales, interdisciplinariedad y paradigma crítico de la historia).

El décimo articulo proviene de Chile y se titula "Perfil de egreso doctoral: una propuesta desde el análisis documental y las expectativas de los doctorandos", de Karen Núñez-Valdés y José Alejandro González Campos. Abordan la discusión entre las expectativas de los estudiantes de doctorado con respecto a las competencias que debiesen adquirir tras su formación. La investigación desemboca en una propuesta para la elaboración de un perfil de egreso estándar para los programas de doctorado en educación.

En el trabajo "Nivel de satisfacción con la estrategia de investigación científica en una facultad de la Universidad Nacional de Chimborazo, Ecuador", de Yosbanys Roque Herrera, Santiago Alonso García y Ana Elizabeth Maldonado León, se presentan los resultados de un estudio cuyo propósito consistió en determinar el nivel de satisfacción de los docentes involucrados en la implementación de una estrategia de investigación científica e innovación tecnológica en la Facultad de Ciencias de la Educación, Humanas y Tecnologías de la Universidad Nacional de Chimborazo. El diagnóstico ofreció los elementos del diseño de una estrategia de conducción y ejecución del proceso de investigación. 
En el decimosegundo lugar aparece el trabajo de Nancy Barragán Machado, Iram Isaí Evangelista Avila y Paola Margarita Chaparro Medina titulado "Una interpretación desde la perspectiva de la hermenéutica analógica: la educación basada en la convivencia", el cual tiene como objetivo mostrar ciertos lineamientos de la educación basada en la convivencia y algunos de los problemas que se presentan cuando se intenta implementar en una cultura que privilegia la competencia. El estudio concluye que una visión analógica permite otorgar claridad en cuanto a las necesidades que deben conciliarse en la educación: aprender habilidades técnicas para un desempeño en el mundo actual y aprender habilidades socioemocionales centradas en el trabajo en equipo, la cooperación, el respeto y el afecto por los demás.

El último artículo es de la autora argentina Míriam Piani Mailhos y se titula "Huellas biográficas de experiencias educativas en la conformación de subjetividades políticas". La investigación se sitúa en instituciones de Bariloche (Patagonia, Argentina) y parte de la relación que existe entre el proyecto colonizador occidental y los procesos educativos a partir de una perspectiva decolonial en diálogo con categorías teóricas críticas; se indaga en las experiencias educativas de docentes, estudiantes y graduados, recuperando huellas de colonizaciones (maneras de ser-estar hacer en el mundo y de conocer).

Finalmente, en la sección de reseñas, Benjamín Salvador Simón analiza "Cultura y educación para la paz. Una perspectiva transversal", un esfuerzo de dos investigadores que llevan la cultura y la educación para la paz como bandera y que lo demuestran con sus acciones diarias, tanto profesionales como personales. En el libro se maneja a la perfección la materia desde una perspectiva tanto teórica como práctica, haciendo que se trate de una obra liviana a la lectura a pesar de exponer al lector la síntesis de pensamientos e ideas complejas, lo cual halaga las aptitudes analíticas, de síntesis y de divulgación del conocimiento que poseen los autores, que cautivan al lector en cada párrafo.

Con esta entrega, y parafraseando de nueva cuenta a Latapí (2008), consideramos que desde la Red de Investigadores Educativos Chihuahua AC realizamos esfuerzos importantes para que la investigación se convierta en herramienta para superar las adversidades educativas. Tenemos fe en que nuestros esfuerzos contribuyen para lograr un país más justo y más humano, y esa fe se alimenta en un horizonte utópico que se traduce en esperanza.

\section{ReFrerevolis}

Casanova Cardiel, H. (2018, octubre 11). La política educativa de cara al nuevo sexenio. ¿Qué reformar de la reforma constitucional de 2013? Mesa panel durante el IV Congreso Internacional de Investigación Educativa en Chihuahua. Chihuahua, México: Red de Investigadores Educativos Chihuahua.

Congreso de la Unión. (2002). Ley de ciencia y tecnología. México: Cámara de Diputados del Congreso de la Unión.

Gil Antón, M. (2018, noviembre 17). ¿Sistema Nacional de Publicadores? Recuperado de https://www.eluniversal.com.mx/articulo/manuel-gil-anton/nacion/sistema-nacionalde-publicadores

Latapí Sarre, P. (2008). ¿Recuperar la esperanza? La investigación educativa entre pasado y futuro. Revista Mexicana de Investigación Educativa, 13(36), 283-297.

López, Sañudo y Maggi. (2013). Investigaciones sobre la investigación educativa 20022011. México: COMIE, ANUIES. 